\title{
El català parlat pels jueus de Cervera a la segona meitat del segle XV
}

\author{
Josep M. ${ }^{\text {a Llobet I Portella * }}$
}

\section{MOTIVACIÓ I OBJECTIU}

A diferència de la llengua castellana que, més o menys alterada, s'ha conservat entre alguns descendents d'aquells jueus que foren expulsats dels regnes hispànics l'any 1492, el català sembla que no ha deixat rastres fàcilment perceptibles en la llengua de les actuals famílies sefardites. Amb tot, ino seria possible, en cas que aquells jueus establerts a les poblacions catalanes coneguessin i usessin la llengua catalana, detectar algunes paraules o construccions d'aquell català antic en la parla actual dels jueus 0 , almenys, en escrits no catalans posteriors al segle $\mathrm{XV}$, que mostressin l'origen català de l'emissor del missatge?

Abans, però, és necessari saber quin era el grau de domini del català que tenien els membres d'aquella generació de jueus establerts a les poblacions catalanes que, en la seva major part, van haver d'abandonar el país durant l'any 1492 .

L'objectiu d'aquest treball és, doncs, donar un primer pas en aquest sentit $i$, en concret, oferir un recull de frases catalanes usades pels jueus habitants a Cervera ${ }^{\dagger}$ durant la segona meitat del segle $\mathrm{Xv}$, amb una doble finalitat: en primer lloc conèixer el grau de domini de la llengua que tenien aquelles persones i, posteriorment, possibilitar la detecció de formes escrites o parlades d'origen català usades pels jueus del mateix origen després de l'expulsió de $1492^{2}$.

* UNED. Centro Asociado de Cervera.

La ciutat de Cervera és la capital de la Segarra, comarca situada a l'interior de Catalunya. Hom trobarà dades geogràtiques i històriques sobre aquesta població a: Gran geografia comarcal de Catalunya, vol. 9. Barcelona, Fundació Enciclopèdia Catalana, 1983, pàgs. 44-65.

2 Pel que fa a l'expulsió dels jueus de Cervera (i també de Tàrrega, població que dista 12 km de l'anterior): LLobet I Portella, Josep M., "Els jueus de Cervera i Tàrrega, l'any 1492", 


\section{METODOLOGIA}

Si bé els documents cerverins escrits en català i procedents de persones jueves són abundants, no hi ha seguretat que fos el mateix interssat el que escrivís el text, raó per la qual aquests escrits no són vàlids per al nostre objectiu. Sortosament, disposem d'un altre tipus de documents en els quals un notari escrivia exactament allò que manifestava oralment la persona jueva, cosa que ens permet conèixer amb més garantia el lèxic $i$ les construccions usades per la persona que parlava. En conseqüència, hem portat a cap una recerca sistemàtica entre la documentació notarial produïda a Cervera durant la segona meitat del segle $\mathrm{XV}^{3}$.

El resultat ha estat la localització de trenta-quatre textos ${ }^{4}$, amb els quals hem elaborat els apèndix I i II. En el primer oferim la transcripció dels documents. En el segon, donem, per ordre alfabètic, el vocabulari procedent de les frases pronunciades per persones jueves. Un tercer apèndix conté la nòmina dels jueus esmentats en la documentació.

\section{CONCLUSIONS}

L'anàlisi de les expressions usades pels jueus cerverins durant la segona meitat del seglexv que es troben contingudes en els documents transcrits, ens permet afirmar que el grau de domini que tenien de la llengua catalana devia ser similar a la resta dels habitants de la població, ja que gairebé totes les formes es troben recollides com a pròpies del català en les obres especialitzades ${ }^{5}$.

Sembla que no hi ha diferència apreciable, pel que fa al grau de domini de la llengua, entre les formes d'expressió usades pels homes i les de les dones.

Aquest conjunt de mots i construccions, encara que només sigui una petita mostra del català usat pels jueus de Cervera en el període estudiat,

I Col.loqui d'història dels jueus a la corona d'Aragó. Lleida, Institut d'Estudis Ilerdencs, 1991, pàgs. 349-368.

3 Un inventari de la documentació notarial: CANeLA, Montserrat, i Garrabou, Montse, Catàleg dels protocols de Cervera. Barcelona, Fundació Noguera, 1985.

Aquests documents permeten conèixer, a més dels aspectes lingüístics objecte del present estudi, tot un conçjunt de formes de vida pròpies d'aquells cerverins - jueus i cristiansde la segona meitat del segle xv.

5 Ens han estat especialment útils les obres: Alcover, Antoni M. ${ }^{a}$, i MoLL, Francesc de B., Diccionari català-valencià-balear, 10 vols. Palma de Mallorca-Barcelona, 1980; i CoROMINEs, Joan, Diccionari etimològic i complementari de la llengua cataiana, 9 vols. Barcelona, Curial 1980-1991. 
pot ser un útil instrument per ajudar a buscar el rastre d'aquesta llengua entre els descendents d'aquells cerverins expulsats, ara fa gairebé cinccents anys, de llur propi país.

\section{APÉNDIX 1}

DOCUMENTS

1

1455, juny, 13. Cervera.

Pere Montaner, batlle de Montroig, cita lçach de Querci, menor, jueu de Cervera.

Arxiu Històric Comarcal de Cervera, Fons Notarial, Cervera, 45, Agustí Martell, Manual, 1454-1455, foli 71.

Dicto die.

Honorabilis Petrus Muntaner, baiulus ut dixit loci de Monroig, in villa Cervarie personaliter constitutus ante presenciam Içach de Quercí, minoris dierum, iudei dicte ville, dixit eidem hec vel similia verba in effectu: «Sènyer, jo com a balle de Monroig vos cit que dilluns ab continuació de dies sia en lo dit loch de Monroig a veure segar los blats d'en Jacme Rull hi d'en Anthoni Rull. En altra manera, encara que no hi fósseu, jo.ls faré segar, vostra absència no contrastant». De quibus, etc.

Et dictus Içach de Quercí, minor dierum, iudeus, dixit: "Jo que deman trellat del que dieu e que no.m córrege temps fins lo haia hagut». Et hanc, etc.

Testes Bernardus dez Vall, domicellus, dominus in parte de Muntpahó, et lohannes Ponç, notarius Cervarie.

1455, juny, 25. Cervera.

Bartomeu Maçana, batlle de Gàver, cita lçach de Querci, major, jueu de Cervera.

AHCC, FN, Cervera, 45, Agustí Martell, Manual, 1454-1455, foli 72 verso.

Die $X X V^{2}$ dicti mensis iunii.

Bartholomeus Maçana, baiulus ut dixit loci de Gàver pro honorabile domina Saureta, uxore honorabilis Galcerandi de Gàver, quondam, domina dicti loci, existens personaliter constitutus in villa Cervarie coram Içach de Quercí, maiore dierum, iudeo dicte ville, dixit eidem sequencia verba: «Níçach, jo com a balle de Gàver vos cit que vingats en lo loch de Gàver a veure segar, tirar, batre, mesurar hi stogar los blats que són stats assignats per mi a vostre procurador dels béns d'en Bernat 
Vilaplana, Johan Miquel, Jaume Sala e Bernat Ragadell, del dit loch. En altra manera, jo.ls faria segar, tirar, batre, mesurar hi stogar, vostra absència no contrestant. E requir a vós, en notari, me.n leveu carta, etc.». De quibus, etc.

Et dictus Içach de Querci dixit: «Sènyer, designau-me dia a segar hi a tirar hi a batre, mesurar hi stogar, que jo o mon procurador hi serem». Et hanc responsionem, etc.

Et dictus Bartholomeus Maçana, baiulus qui supra, dixit replicando sequencia verba: "Sènyer, jo us designe que hi siau demà ab continuació de dies fins sia segat, tirat, batut, mesurat hi stogat». Quam replicacionem, etc.

Et in hiis fuerunt testes presentes lohannes Maior, blanquerius, et lohannes Puig, alias Barçaló, faber, Cervarie.

1455, juny, 25. Cervera.

Pere Bonet, lloctinent del batlle de Concabella, cita Samuel de Lunell i Jucef Cavaller, jueus de Cervera.

AHCC, FN, Cervera, 45, Agustí Martell, Manual, 1454-1455, foli 73.

Dicta die.

Petrus Bonet, botigerius Cervarie, locumtenens ut dixit Raymundi Sunyer, minoris dierum, baiuli loci de Concabella, existens personaliter constitutus in dicta villa Cervarie coram Samuele de Lunell, iudeo fisicho, ut asseritur tutore et curatore pupillorum filliorum Samuelis Astruch Cavaller, quòndam iudei, et luceff Samarell (sic), iudeo fillio dicti Samuelis Astruch Cavaller, quondam, dixit eisdem hec que sequntur verba: «Senyors, a vós mestre Lunell, com a tudor e curador dels fills d'en Samuel Astruch Cavaller, quóndam, e a vós en Juceff Cavaller, fill [del] dit Samuel Astruch Cavaller, jo, com a loctinent del balle de Concabella, vos cit e us intim que vuy qui és dimecres siau en lo dit loch de Concabella a veure segar; e com sia segat, tirar e batre; e aprés, mesurar e stogar e veure destribuhir los blats que són stats assignats per lo dit balle de Concabella a.n Pere Costa, en nom dels dits pubills fills del dit Cavaller, d'en Ramon Sunyer del dit loch. En altra manera, lo balle farà segar, tirar, batre, mesurar, stogar hi destribuhirà los dits blats, la absència dels dits pubills ni de vós, mestre Lunell, com a tudor de aquells, no contrestant. E requir a vós, en notari, me'n leveu carta». De quibus, etc.

Et dicti Samuel de Lunell, assertus tutor et curator, et luceff Cavaller, fillius dicti Samuel Astruch Cavaller, dixerunt: «Sènyer, a nosaltres plau que los blats del dit en Ramon Sunyer sien segats e tirats, e que ho remetem a fe del balle. Tant com dieu de batre, mesurar, stogar e desíribuir, no y consentim fins nos doneu jornada serta, e lavors lo actor e procurador dels dits pubills hi serà». Et hanc responsionem qui, etc.

(Falten els testimonis).

1455, juliol, 8 . Cervera. 
Pere Jutglar, lloctinent del batlle de Carbasí, cita Cresques Adret, jueu de Cervera.

AHCC, FN, Cervera, 45, Augustí Martell, Manual, 1454-1455, foli 75.

Die martis VIII dicti mensis iulii.

Petrus Jutglar, loci de Quarbesí, locumtenens ut dixit Petri Jutglar, bajuli dicti loci de Quarbesí, existens personaliter constitutus in villa Cervarie, scilicet in domo Cresques Adret, iudei fisici, quam fovet in callo superiori dicte ville, dixit eidem hec que sequntur verba: «Sènyer en Cresques, jo com a loctinent de balle de Quarbesí, vos intim e us cit que demà ab continuació de dies vengau al dit loch a veure segar, tirar, batre hi stogar aquells blats que per mi, com a loctinent de balle del dit loch, vos són stats assignats dels béns meus». De quibus, etc.

Et dictus Cresques Adret respondendo dixit: «A mi plau, sènyer, que tant com toque de segar, tirar hi batre, que son content que los blats que.m són stats assignats sien segats, tirats e batuts, emperò que lo balle ho face fer hi aquells tinge, com sien batuts hi bells, a dret hi justícia per fer paga a mi en mon deute, messions e interessos. Tant com toque al stogar ni en fer altre exercici, de aquí avant no y consint ans lo requir que com seran bells me cit e.m don jornada designada». Et hanc, etc.

Testes Petrus Pintor, ville Guissone, et Petrus Davins, ville Cervarie.

1455, juliol, 11. Cervera.

Ramon Sunyer, batlle de Concabella, cita lçach de Quercí, major, jueu de Cervera.

AHCC, FN, Cervera, 45, Agustí Martell, Manual, 1454-1455, foli 75 verso.

Die veneris $X 1^{a}$ dicti me[n]sis.

Raymundus Sunyer, baiulus loci de Concabella, in villa Cervarie personaliter constitutus, coram Içach de Quercí, iudeo, maiore dierum, dicte ville, requisir, monuit et citavit eundem quatenus intersit die lune cum continuacione dierum ad videndum, tirar, batre, mesurar hi stogar blada que fuerunt sibi assignada per eundem baiulum de bonis Berengarii Lorenç, maioris dierum, dicti loci. Alias, etc. De quibus, etc.

Et dictus Içach de Quercí respondendo dixit: «Sènyer, jo hi trametré mon procurador dilluns, si no que vage a mon carrrech".

Testes discretus Dalmacius Sellés, notarius, et Petrus Quer, pelliparius, Cervarie.

6

1455, juliol, 11. Cervera.

Ramon Sunyer, batlle de Concabella, cita Samuel de Lunell, jueu de Cervera.

AHCC, FN, Cervera, 45, Agustí Martell, Manual, 1454-1455, folio 76. 
Dicta die.

Item dictus Raymundus Sunyer, baiulus predicti loci, in dicta villa personaliter constitutus, monuit et citavit Samuelem de Lunell, fisichum, ut tutorem et curatorem pupillorum filliorum Samuelis Astruch Cavaller, quondam iudei dicte vilie, quatenus die lune proxima venienti cum continuacione dierum intersit in dicto loco de Concabella ad videndum tirar, batre, mesurar hi stogar hi destribuir blada que fuerunt assignata actori pupillorum filliorum dicti Samuelis Astruch Cavaller, quondam, de bonis Berengarii Lorenç, maioris dierum, et Berengarii Lorenç, minoris dierum, dicti loci. Alias, etc. De quibus, etc.

Et dictus Samuel de Lunell dixit: "Sènyer, a mi plau que los blats sien tirants, batuts, mesurats hi stogats, e que aquells tingau a dret. Tant com toque al distribuir, no y consent sens que n.m doneu [jornada] designada, quar jo hi tremetré lo actor». Et hanc, etc.

Testes Gabriel Borreda, agricola, et Galcerandus Rialp, carnifex, Cervarie.

1455, juliol, 30. Cervera.

Pere Ferrer, lloctinent del batlle d'Albarells, cita Cresques Adret, jueu de Cervera, mitjançant Bonafilla, esposa d'aquest jueu.

AHCC, FN, Cervera, 45, Agustí Martell, Manual, 1454-1455, foli 77 verso.

Dicta die.

Item ipsa eadem die dictus Petrus Fferrarii, locumtenens baiuli predicti loci d'Aubarells, existens constitutus in dicta villa, videlicet ante domum Cresques Adret, iudei dicte ville, in qua erat domina quedam apellata Bonafilla, uxor dicti Cresques Adret, dixit eidem domine sequencia verba: "Madona iés hic vostre marit? quar jo son ací com a loctinent del balle d'Aubarells, que.l cit hi amonest, ací en sa habitació, que demà ab continuació de dies sie en lo loch d'Aubarells a veure batre, mesurar hi stogar los blats que són stats assignats per lo dit balle a sos procuradós dels béns d'en Bernat de Sils e d'en Pere Borrell, del mas d'Altalló, terme d'Albarells. Altrament, sa absència no contrestant, los blats siran batuts, mesurats hi stogats». De quibus, etc.

Qui quidem dicta Bonafilla, uxor dicti Cresques, respondendo dixit: «Sènyer, mon marit no és en vila, mas jo ho diré als seus procuradós». Etc.

Testes lohannes Fuster, agricola Cervarie, et Ffrancischus Carnicer, apothecarius ville Tarrege.

8

1455, agost, 14. Cervera.

Pere Jutglar, lloctinent del batlle de Carbasi, cita Cresques Adret, jueu de Cervera, mitjançant Bonafilla, esposa d'aquest jueu.

AHCC, FN, Cervera, 45, Agustí Martell, Manual, 1454-1455, foli 78 verso. 
Die iovis XIIII dicti mensis augusti.

Petrus Jutglar, locumtenens ut dixit Petri Jutglar, baiuli loci de Quarbesí, in villa Cervarie, videlicet in domo Cresques Adret, iudei dicte ville, personalites constitutus, coram domina Bonafilla, uxore dicti Cresques, dixit eidem domine hec que sequntur verba: "Madona iés hic vostre marit?"

Que quidem domina dixit: «No ych és en vila».

Et dictus Petrus Jutglar dixit: «Veus, madona, que jo, com a loctinent de balle de Quarbesi, cit vostre marit que dilluns primer vinent sie a Quarbesi a veure mesurar hi stogar los blats que per lo balle són stats assignats dels béns meus e, axí mateix, que hi sie al dit dia a distribuir. Altrament, si ell no y ere, los blats seran mesurats, stogats hi distribuïts, etc.» De quibus, etc.

Testes Matheus Bonjoch et Petrus Jover, loci de Monleó, christiani, et Lobell Samarell, iudeus Cervarie.

1455, octubre, 7. Cervera.

Guillem Armengou, lloctinent del batlle de Massoteres i altres llocs, cita Jacob de Querci, jueu de Cervera.

AHCC, FN, Cervera, 45, Agustí Martell, Manual, 1454-1455, foli 84 verso.

Die martis $\mathrm{VII}^{\mathrm{a}}$ mensis octobris, anno $\mathrm{M}^{0}$ CCCC $\mathrm{LV}^{0}$.

Guillemus Armengou, loci de Palou de Taltaüll, in villa Cervarie personaliter constitutus, locumtenens ut dixit lohannis Pintor, baiuli locorum de Maçoteres, de Taltaüll, de Palou e de Cornudella, coram lacob de Quercí, iudeo dicte ville, intimavit que demà ab continuació de dies sie a veure venemar hi encubar la venema que és stada assignada a son procurador dels béns d'en Jacme Johan e d'en Anthoni Pla, del dit loch de Cornudella. E, axí mateix, a veure cullir, sbrinar, acolrar e levar de les posts los safrans que li són stats assignats dels demunt dits. $E$ aço sens preiudici de la sentència donada entre los pagesos e los juheus. Alias protestatus fuit, etc. De quibus, etc.

Et dictus lacob de Quercí dixit: «Sènyer, jo que no consint en vostra citació com no.m haiau citat als blats». Et hanc, etc.

Testes Bartholomeus Bermon, de la Amella, Anthonius Lenyedor, christiani, et luceff Azfor, iudeus Cervarie.

1455, octubre, 7. Cervera.

Guillem Armengou, lloctinent del batlle de Massoteres i altres llocs, cita lçach de Querci, major, jueu de Cervera.

AHCC, FN, Cervera, 45, Agustí Martell, Manual, 1454-1455, foli 86. 
Dicta die.

Guillemus Armengou, loci de Palou de Taltaüll, locumtenens lohannis Pintor, baiuli locorum de Maçoteres, de Taltaüll, de Palou e de Cornudella, in villa Cervarie personaliter constitutus, coram Içach de Quercí, maiore dierum, iudeo Cervarie, intimavit que demà que serà dimecres venge a veure venemar hi encubar la venema que és stada assignada a son procurador. E, axí mateix, a veure cullir, sflorar, acolrar e levar de les posts lo saffrà que és stat assignat al dit son procurador dels béns d'en Anthoni Pla e Jacme Johan, de Cornudella, Berenguer Giner e Bernat Cortades, de Maçoteres, Leonart Mulnell, de Palou, Guim Armengou, del ditloch de Palou, e Johan Castellar, de Taltaüll. E açò sens preiudici de la sentència que s'és donada entre los juheus e los patgesos. Alias protestatus fuit, etc. De quibus, etc.

Et dictus Içach de Quercí, maior dierum, dixit: "Sènyer, a mi plau que lo saffrà dels demunt dits sie cullit e acolrat e levat de les po[s]ts, emperò que venga en poder del balle. E més me plau que la venema dels demunt dits sie venemada e encubada, salvant-me dret, emperò, en los actes que hi he fets e de marcha que $y$ entench haver». Et hanc, etc.

Testes discretus lohannes d'Aubalat, notarius Cervarie, et Gilius Spluga, Ioci de Thorano.

1455, novembre, 12. Cervera.

Pere Jutglar, lloctinent del batlle de Carbasi, cita Cresques Adret, jueu de Cervera.

AHCC, FN, Cervera, 45, Agustí Martell, Manual, 1454-1455, foli 93 verso.

Die XII dicti mensis novembris.

Petrus Jutglar, locumtenens ut dixit Petri Jutglar, baiuli loci de Quarbesí, in villa Cervarie personaliter constitutus, coram Cresques Adret, iudeo fisicho Cervarie, dixit eidem sequencia verba: «En Cresques, jo us cit que vejau venemar aquella sort de venema que per mi, com a loctinent de balle, vos és stada assignada, a demà, que serà digous, ab continuació de dies». De quibus, etc.

Et dictus Cresques Adret dixit. «Sènyer, la venema ja és venemada de què sou caygut en marcha perquè no.m haveu citat al venemar e a altres coses". Et hanc, etc.

Et dictus Petrus Jutglar, locumtenens predictus, dixit: «Sènyer, anau-hi e veureu com [no] és venemada». Et predictam replicacionem, etc.

Testes Petrus Soldevila, de Granyena, Bernardus Figera, de Muntesquiu, et luceff Samarell, iudeus Cervarie.

1455, desembre, 5. Cervera.

Pere Borredà i Francesc Planich, campaners de Cervera, comuniquen a Içach de Querci, menor, i Salamó Satorra, secretaris de l'aljama dels jueus de Cervera, una cessió de diners. 
AHCC, FN, Cervera, 45, Agustí Martell, Manual, 1454-1455, foli 98.

Die veneris $V^{a}$ dicti mensis decembris.

Petrus Borredà et Ffrancischus Planich, campanerii ville Cervarie, in eadem villa Cervarie personaliter constituti, coram Içach de Quercí, minore dierum, et Salamone Çatorra, iudeis segretariis anno presenti aliame iudeorum dicte ville, dixerunt eisdem hec vel similia que sequntur: "Senyors de segretaris, nosaltres, de aquelles vinthi-set lliures e mitga barchinonenses, les quals nosaltres havem de soldada per regir lo campanar de aquesta vila e les quals los juheus de la aljama de aquesta vila són tenguts donar a nosaltres, e com nosaltres de les dites XXVII lliures e mitga haiam fetes loch hi cessió al discret mossèn Jacme Albesa, prevere, ab carta presa per lo discret en Sadurní Planes, notari d'aquesta vila, vos diem e us requerim, com a segretaris demunt dits que sou, que de les dites vint-hi-set lliures e mitga respongau e façau responre al dit mossèn Albesa o a qui ell volrà, d'aquí avant, axí com a nosaltres ereu tenguts abans del loch hi cessió per nosaltres a ell fet”. De quibus, etc.

Et dicti Içach de Quercí et Salamo Çatorra segretarii predicti, dixerunt: «Nosaltres, senyors, som contents de responre del que.ns intimau en aquell qui justícia volrà hi ordenarà». Et hanc, etc.

Testes discretus lohannes Morell, prebiter, et Bernardus Robió, sartor, ville predicte Cervarie habitatores.

1456, juliol, 15. Guimerà

lçach Adret, administrador de l'aljama dels jueus de Cervera, intima a Jaume Molner, agricultor de Guimerà, noves disposicions sobre la lleuda de/s jueus forasters.

AHCC, FN, Cervera, 46, Antoni Busquets, Manual, 1455-1456, foli 70.

Die $X^{a}$ iulii dicti anni a nativitate Domini $M^{i} \operatorname{CCCC}^{i} L^{i}$ sexti.

Noverint universi [espai en b lanc] Içach Adret, iudeus ville Cervarie, administrator ut dixit aliame sive iudeorum ville Cervarie, existens personaliter in loco de Guimerano, ante presenciam mei notarii et testium infra scriptorum specialiter ad hech vocatorum et assumptorum, et seu coram lacobo Molner, agricola dicti loci, dixit eidem lacobo hech verba vel istis similia verba: "Sènyer, io us dich hi us intim que de si avant vós no responau a nengú de la leuda dels juheus strangers pasants per aquest leudari, sinó tant solament a mi o al qui serà administrador de la dita almoyna, o a qui nosaltres voldrem».

Et dictus lacobus dixit verbo que ell ho té arendat e que ell ho cullirà e que, après, ell ne donarà rahó a qui donar la degue, però aquel que li'n ha arendat li'n starà al davant, e que de qui avant és prest de respondre'n al dit juheu administrador ho a qui voldrà.

Et de facto dictus Içach Adret, dicto nomine, creavit in receptore dicte lezde dictum lacobum Molner. Qui quidem lacobus Molner prestit iuramentum [...]. 
Testes honorabiles Iohannes Alegre, baiulus loci de Guimerano, et Bonifacius Biri (?), familiaris de domo nobilis laufridi de Castro.

Actum Guimerano.

1457, febrer, 18. Cervera.

Joan Gibert, procurador de Jaume Serró, prevere de Cervera, demana a Mossé Sutlam, jueu de Cervera, la devolució d'una tassa d'argent.

AH, FN, Cervera, 45, Agustí Martell, Manual, 1457-1459, foli 6.

Die XVIII ${ }^{a}$ dicti mensis ffebroarii.

Iohannes Gibert, causidicus, procurator ut asservit discreti lacobi Serró, prebiteri Cervarie, existens personaliter constitutus coram Mosse Sutlam, iudeo dicte ville, dixit eidem hec vel similia ver[b]a: «En Mossé, vós teniu una taça d'argent, penyora de mossèn Jacme Serró, prevere de aquesta vila, per trenta-nou sous, per què jo son prest hi aparellat de pagar-vos ab que.m torneu la dita taça, hi no stà per mi sinó per vós que aquella de veres vós voleu retenir. $E$, per maior descàrrech, vos offir ací e us mostre que.s donaré tres florins d'or. En altra manera, protest de pèrdua de la dita taça e de tots dans [e] despeses. E requir a vós, en notari, me'n leveu carta». Et de facto coram dictis testibus mostravit sibi tres florenos auri. De quibus, etc.

Et dictus Mose Sutlam respondendo dixit: «Sènyer, jo son content de tornar-laus a vós o a vostre principal d'aquesta setmana qui ve, quar ara no poria, que penyora la he mesa». Et hanc, etc.

Qui Gibert dixit quod non consentiebat ymo protestabat, etc.

Testes Matheus Bonjoch, agricola, et Iohannes Romeu, questor, Cervarie.

1458, gener, 13. Cervera.

Guillem Armengou, lloctinent del batlle de Talteüll, cita Jucef Cavaller, jueu de Cervera.

AHCC, FN, Cervera, Agustí Martell, Manual, 1457-1459, foli 38.

Die veneris XIII dicti mensis.

Guillermus Armengou, loci de Palou, termini castri de Taltaüll, locumtenens ut dixit lohannis Jener, baiuli castri et termini de Taltaüll, existens personaliter constitutus in villa Cervarie, coram luceff Cavaller, iudeo dicte ville, dixit eidem sequencia verba: "En Juceff Cavaller, jo, com a loctinent del honorable en Johan Giner, balle del castell e terme de Taltaüll, vos cit e us intim que digous qui ve, que comptarem

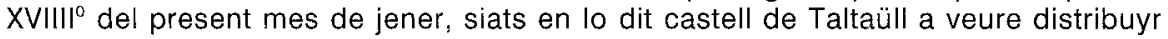
los béns que a instància vostra són stats emparats dels béns d'en Bernat Palou, 
Leonart Mulnell, Guillem Armengou, de Palou, e d'en Bernat Castellar, de Taltaüll. En altra manera, vostra absència no contrestant, los dits béns per vós emparats seran destribuyts».

Et dictus luceff Cavaller respondendo premissis dixit: «Sènyer, jo ignor que vós siats loctinent de balle del castell e terme de Taltaüll».

De quibus omnibus dictus Guillermus Armengou requisivit instrumentum, etc.

Testes lohannes Robió, sartor, Iohannes Ferriça, asaunator, christiani Cervarie, et Isach de Besés, iudeus Tarrege.

1458, juliol, 14. Cervera.

Jaume Peiró, batlle de la Curullada, cita Jucef Cavaller, jueu de Cervera.

AHCC, FN, Cervera, 43, Joan Ponç, Manual, 1457-1458, foli 36 verso.

Die XIIII iulii dicti anni.

lacobus Peyró, baiulus loci de la Curullada, constitutus personaliter in villa vestra (sic), domo luceff Cavaller, iudei Cervarie, verbo citavit dictum luceff cum exposicione: «A veure tirar, batre, mesurar e distribuir los blats d'en Anthoni Guerau e d'en Guillem Soler e de mi, dit Jachme Peyró, los quals en virtut de certes fadigues de mossèn lo veguer de Cervera vos són stats assignats".

Cui quidem citacioni dictus Cavaller taliter duxit respondendum: «A mi plau que tiren los blats assignats, però vull que.m sie donada jornada a batre, car yo só prest de anar-hi o de trametre-y. E vós, en notari, continuam'o axís.

Et sich dictus baiulus assignavit dicto iudeo diem lune proximem a veure batre.

Dictus vero iudeus dixit: "No.m plau lo tirar de disapte".

De quibus instrumentum publicum, etc.

Testes sunt Johan Robió, sartre, e Pere Ramon, ma[n]yà, [e] Juceff Baró Crexent, iudeus, Cervarie.

1459, febrer, 16. Cervera.

Felip Prats, regent de Montoliu, cita Jucef Cavaller, jueu de Cervera, mitjançant Goig, mare d'aquest jueu.

AHCC, FN, Cervera, 45, Agustí Martell, Manual, 1457-1459, foli 97 verso.

Die $X V I^{a}$ dicti mensis ffebroarii. 
Religiosus frater Philipus Prats, ordinis Sancti Bernardi, regens locum de Muntuliu pro reverendo abbate Sanctarum Crucum, in villa Cervarie personaliter constitutus, coram dicta Goig, uxore Samuelis Cavaller, quondam iudei dicte ville, dixit eidem domine Goig hec verba: «Digau ¿és hic vostre fill en Juceff Cavaller?»

Que domina dixit: "No hic és».

Qui maioral dixit: «Per ço lo deman, quar jo, com a maioral del dit loch de Muntuliu, lo cit ací, hon té sa habitació, per tant com dieu que no hic és, que sie dilluns qui ve al dit loch per veure destribuyr lo preu del aiberch que ere de Johan Rossell, quòndam del dit loch, lo qual preu és stat emparat per lo dit Juceff, del qual preu és stat fet depòsit. En altra manera, sa absència no contrestant, lo dit preu serà distribuyt». és".

Que domina dixit: «Jo no consent en vostra citació per tant com mon fill no hic

De quibus, etc.

Testes Petrus de Cornellana, notarius, et lohannes Nadal, curritor auris, et luceff Baró Crexent, iudeus, Cervarie.

1459, agost, 16. Cervera.

Joan Barulls, batlle de Vicfred, cita Jacob de Querci, jueu de Cervera.

AHCC, FN, Cervera, 46, Antoni Busquets, Manual, 1458-1459, foli 41 verso.

Die et anno proxime scriptis.

Iohannes Barulls, baiulus loci de Bicfret, constitutus in villa Cervarie, citavit lacob de Quercí, iudeum ville Cervarie, dicendo eidem hec verba vel istis consimilia in effectu: «Sènyer en Jacob, jo us scit que dins deu dies primers vinents vós siau ho tremetau vostre procurador en lo loch de Bicfret per veure distribuhir los blats per vós emparats d'en Ramon Johan del dit loch. En altra manera que procehiré a la execcució de aquels, segons que per justícia trobaré ésser fahedor he mon assesor me consellarà. Requerint a vós en notari me.n fasau carta, etc.».

Et dictus lacob de Quercí premissis respondendo dixit verbo: «Jo que no y iria ni y trametria sinó que declararé una marcha".

De quibus, etc.

Testes discretus Ffranciscus Saliteda, notarius Cervarie, et Berengarius Miret, loci de Briançó, vocati, etc.

Actum Cervarie.

1460, juny, 25. Cervera.

lçach de Querci, jueu de Cervera, requereix a Pere Macià, agricultor de Santa Fe,

el pagament de certa quantitat. 
AHCC, FN, Cervera, 46, Antoni Busquets, Manual, 1460, foli 34 verso.

\section{Die $X X V^{a}$ iunii.}

Içach de Quercí, iudeus ville Cervarie, constitutus personaliter in villa Cervarie et in vico maiori, etc., [espai en blanc] coram Petro Macià, agricola loci de Senta $\mathrm{Fe}$, personaliter reperto verbo dixit, etc.: “Jo que us requir, sènyer en Macià, que.m pagueu dins spay de cinch jorns tot Cervarie, procurator ut aservit et nomine et pro parte [espai en blanc] obtulit et presentavit et per me eundem notarium legi peciit et requisivit, coram Bernardo Colomer et lçach Sutlam, iudeo, Cervarie habitatoribus, repertis in vico maiori, ante curiam dechanatus Cervarie, procuratoribus Salamonis Coffén, iudeo (sic) Cervarie olim nunch vero civi (sic) Illerde, quandam papiri patentem litteram serenissimi domini regis Aragonum et Castelle eiusque sigillo in dorso sigillatam, cum signo de registrata, huiusmodi seriey: «Fferdinandus, Dei gracia rex Castelle...». Inseratur.

Qua lecta continuo dictus Bernardus Colomer peciit copiam pro testibus, etc., que fuit sibi concessa et per me tradita, et dictus Içach Sutlam dixit: "No gos responre ni dir res, que vuy és dissapte».

De quibus, etc., instrumentum et instrumenta, etc., tot quot, etc.

1484, gener, 28. Cervera.

Salamó Cohen, jueu habitant a Cervera, requereix a Cresques Adret, un dels secretaris de l'aljama dels jueus de Cervera, i a lçach Jacob de Quercí, lloctinent de secretari, que disposin la celebració immediata d'un consell.

AHCC, FN, Cervera, 53, Antoni Bonet, Manual, 1483-1484, foli 73.

\section{Die XXVIII ${ }^{2}$ mensis ianuarii, anno $M^{0}$ CCCC LXXXIIII.}

Dicta die in presencia mei Anthonii Bonet, ville Cervarie, regia auctoritate notarii publici infra scripti, et in presencia eciam Vitalis Simeon, Sancte Columbe de Queralto, iudei [et] Abram Azcarell, iudei illerde civitatis, testium, etc., Salamo Coffén, iudeus civitatis Illerde, in villa Cervarie degens, constitutus intus quoddam studium domus magistri Cresques Adret, que est in callo superiori dicte ville, circa horam nonam ante meridiem, verbo potulit talia verba: "Continua-me en notari com yo aga request de paraula als secretaris tinguessen consell e no.n agen volgut fer. Ara requer a mestre Cresques Adret, secretari, e a mestre Içach lacob de Querci, loctinent de secretari, de la aliama dels jueus de Cervera, que ací són presents, que, en virtut del sagrament prestat per ells, de continent me ajusten consell, com sie perill en la triga. En altra manera, proteste de la fricció del jurament e de tots danys e totes coses lícites de protestar". De quibus, etc.

Et continuo dictus Cresques Adret ut secretarius predictus respondendo premissis sic ait que no li és stat dit ab instància fins ara, que són cerca de nou hores, e encara no ere levat a set e miga, e que ara no és hora còngrua de plegar consell com los demés són manestrals e n'i ha que són fora vila, més, encontinent que haurà oportunitat ni tal necessitat, serà prest. E maravelle's del dit Salamó Cofén que moltes vegades ha request tinguessen consell sobre lo seu fet, e axí és stat 
fet, a feta certa conclusió, e serie fort que tots jorns e totes hores ell pensàs ni pens que li agen haver consell.

Et dictus magister Içach lacob de Quercí dixit que per fer content a dit Salamó e per guardar lo profit e onra de son frare, on que no pogués ésser forçat de usar de la loctinença, segons havie consell de bon juriste, ab tot, anperò, ho vol acceptar en lo seu fet, e que farà tot quant per justícia sie tengut fer, a consell del advocat de la aliama.

Et dictus Salamo Cofén replicando dixit a la resposta del dit mestre Cresques que, ab honor parlant, cesse ésser ver lo per ell posat, ans etén aprovar com ha cerca de dues hores que.l ha request en temps que tots los del consell eren en la scola plegats e per dit Salamó li fou dit com lo consell se deramave e que en la triga havie gran perill e, encara, com a requerén, segons damunt.

Et dictus Cresques ait lo dit Salamó Cofén pot dir lo que.s vol, com ell dit Cresques no és stat a la scola vuy al offici pur cert és, del sol exit, que encara ell no ere levat, fins a la hora que ell matex diu no y havie tal distància, e stà $e$ persevere en lo que dit ha.

De quibus, etc., instrumentum et instrumenta, etc.

1485, agost, 30. Cervera.

lçach Jacob de Querci, jueu de Cervera, requereix a Jucef Cavaller, un dels secretaris de l'aljama dels jueus de Cervera, mitjançant Bonadona, esposa del secretari esmentat, que disposi la celebració immediata d'un consell.

AHCC, FN, Cervera, 53, Antoni Bonet, Manual, 1484-1486, foli 77.

Dicta die in presencia mei Anthonii Bonet, ville Cervarie, auctoritate regia notarii publici infra scripti, et in presencia eciam Gabrielis Urgell, sartoris, luceff Baró Crexent et Boniua Salom, iudeorum, Cervarie, testium, etc., Içach lacob de Quercí, ffisicus iudeus dicte ville, constitutus personaliter ante domum luceff Cavaller, iudei, que est in vico maiori eiusdem ville, verbo pecuiit uxori dicti Cavaller si dictus vir suis erat in domo. Et dicta eius uxor Bonadona dixit que non erat in villa. Et ilico dictus Içach de Quercí dixit: «Senyors, feu-me testimoni com yo de paraula vuy aga dit e request a mestre Juceff Cavaller que ell, com a secretari que és, vingués a celebrar consell e tenir consell per feynes de la aliama, specialment per un[a in]tima que és stada feta en virtut de una provisió per ell novament obtenguda del il.lustríssimo senyor infant, e no sie volgut venir, que ara requir ací, a la sua habitació, com la cuyta sie gran, que ell vingue per tenir e celebrar consell, e vós en notari feu-me'n carta».

Et statim Bonadona, uxor dicti luceff Cavaller, dixit que dictus eius vir non erat in villa e com serà vengut ella lo y dirà.

De quibus, etc., instrumentum et instrumenta, tot quot, etc. 
lçach Jacob de Querci, un dels secretaris de l'aljama dels jueus de Cervera, demana a Joan de Llobets, lloctinent del veguer i batlle de Cervera, autorització per reunir el consell de l'aljama.

AHCC, FN, Cervera, 53, Antoni Bonet, Manual, 1484-1486, foli 77.

Eisdem die et anno in presencia mei Anthonii Bonet, notarii, et in presencia eciam venerabilium Iacobi Albesa, lacobi Cornellana, prebiterorum, et Iohannis Cornellana, notarii, testium, etc., Içach lacob de Quercí, iudeus secretarius anno predicto aliama iudeorum dicte ville, constitutus personaliter coram honorabile lohanne de Lobets, locumtenente honorabilis vicarii et baiuli dicte ville, ante scribaniam curie decanatus Cervarie reperto personaliter, verbo dixit et exposuit coram ipso hec vel similia verba in effectu: "Mossèn molt magnífich, sobre la intima que.ns és stada feta per la provisió que mestre Juceff Cavaller ha obtenguda del senyor infant, yo volia ajustar consell e vuy ho diguí a mestre Juceff Cavaller, secretari, e ara jo n'e feta requesta a sa casa e ell és absent, per què, mossèn, vos deman licència de ajustar lo consell de la aliama una e moltes voltes a fi que sobre açò puixam provehir e lo dit consell puixe deliberar e acordar com li parrà».

Et in continenti dictus honorabilis locumtenens dedit et contulit licenciam et plenariam facultatem eidem Içach lacob de Quercí congregandi et congregari faciendi dictum consilium ut est moris et deliberandi super petitis prout eidem consilio videbitur.

De quibus, etc., instrumentum et instrumenta, etc., tot quot, etc.

1488, desembre, 17. Cervera.

Jacob lçach de Querci i Bonet Bellsom, secretaris de l'aljama dels jueus de Cervera, requereixen a Dídac d'Avellaneda la devolució d'una sarbatana.

AHCC, FN, Cervera, 53, Antoni Bonet, Manual, 1487-1488, foli 97.

Die mercuri $X V I^{a}$ mensis decembris, anno predicto a nativitate Domini $\mathrm{M}^{0} \mathrm{CCCC}^{0}$ LXXXVIII

Dicta die in presencia mei Anthonii Bonet, notarii infra scripti, et in presencia eciam honorabilium Iohannis Soldevila, subbaiuli, et Mathei Janer, cerdonis, Cervarie, testium, etc., magister lacob Içach de Quercí, fisicus, et Bonet Bellsom, iudei secretarii aliame iudeorum Cervarie, constituti personaliter in intratus domus magnifici Didaci de Avellaneda, que est in vico maiori, ad vocem dicti Quercí dixerunt et exposuerunt, coram dicto magnifico Didacho de Avellaneda ibidem personaliter reperto, hec vel similia verba in efectu: "Mossèn molt magnífich, a nosaltres és stada presentada una letra de la magestat del senyor rey, closa, ab son segell secret, per lo magnífic mossèn Johan Aymerich, cavaller, en poder del discret n'Anthoni Saliteda, notari, la qual vos presentam». Que littera erat operta et dissigillata sigillo secreto ut prima facie apparebat, in qua ab extra tale erat epigrema: "A la aliama e jueus de la nostra vila de Cervera». Ab intra erat huiusmodi tenoris: "Lo 
rey. Aliama, sabut havem teniu una çarabatana que en lo temps que Avellaneda tenia lo castell li prestàs e perquè la havem mester per al dit castell vos diem e manam molt stretament e sots incorriment de la ira e indignació nostra que, vista la present, la'ns presteu per al dit castell, hon vos serà ben guardada, e si no la teníeu la cobreu, cerficant-vos que no us admetrem excusa alguna. Data en Valladolit, a XXVII de noembre, any M CCCC LXXXVIII. Post data: Liurau la dita çarabatana a nostre creat e alcayt del dit castell mossèn Johan Aymerich ab sos servidors, a qui scrivim la cobre e guarde per vosaltres. Data ut supra. Yo el rey. Ludovicus Gonçales, secretarius». "A la qual presentació havem respost: "Obeynt los manaments reals, som contents prestar dita çarabatana". E perquè vós, mossèn, teniu dita çarabatana, vos requirim la'ns doneu perquè la puixam liurar al dit mossèn Aymerich, al qual la volem leixar en virtut de la dita letra del senyor rey».

Et in continenti dictus magnificus Didachus de Avellaneda respondendo premissis dixit: «Yo deman translat de tot lo que demanau e dieu e protest que no.m corregue temps fins que.I aga agut».

De quibus, etc., instrumentum et instrumenta, etc.

1489, febrer, 21. Montmaneu.

Jafudà de Querci, jueu de Cervera, cita Guillem Soler i Joan Maçart, agricultors de Montmaneu.

AHCC, FN, Cervera, 57, Pere Ponç, Manual, 1487-1489, foli 49 verso.

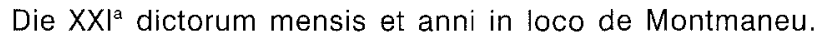

laffuda de Quercí, iudeus ville Cerverie, personaliter constitutus in loco de Montmaneu, coram Guillermo Soler, agricola dicti lloci, citavit et monuit dictum Soler quatenus eidem solveret inffra quinque dies iuxta formam obligacionis per se eidem facte totum id quod sibi debet, alioquin teneret hostaticum personale intus villam Cervarie. De quibus, etc.

Et dictus Soler dixit: «No us cal per axò, que tantost sereu pagat».

Similem citacionem fecit dictus Quercí in habitacione lohannis Maçart, agricole dicti lloci, ipso absente sed uxore sua presente, videlicet dicendo as hostium dicte domus: «És ací lo sènyer en Johan Maçart?"

Et dicta uxor dicti Maçart respondendo dixit: “No y és, deffora és».

Et dictus Quercí dixit: «Ara, madona, digau-li com yo.I requir ab notari, que ací és, que.m pach tot lo que.m deu dins $V$ dies, iuxta forma de la obligació. En altra manera, que tingue hostatges dins Cervera, iuxta forma de aquella».

De quibus, etc.

Testes honorabiles Ffranciscus Tarroga, argentarius, et Nicholaus [espai en blanc]. 
1490, juny, 30. Cervera.

Bonjua Adret, major, jueu de Cervera, denuncia l'obra nova que fa fer Jucef Baró Crexent.

AHCC, FN, Cervera, 55, Antoni Romeu, Manual, 1489-1490, foli 87.

Die mercurii XXX iunii anno predicto Cervarie.

Presente et vocato me Anthonio Romeu, ville Cervarie, auctoritatibus apostolica, imperiali et regia notario publico, constitutus personaliter Boniua Adret, judeus, mayor dierum, Cervaire, in cayllo inferiori dicte ville, coram domibus luceff Baró Crexent, iudei dicte ville, coram quibus faciebat dictus Baró fodere terram per quendam dictum lohannem de Berri, dixit: «Jo denunciu obra nova ací en lo que fa obrar sènyer en Baró e en senyal de dita obra nova, la qual denunciu, lans ací tres pedres, denunciant tres vegades: obra nova, obra nova, obra nova, e requer a vós en Johan de Berri que us ne lexeu, e requer a vós en notari ne leveu carta pública». Quibus, etc.

Testes sunt Michael Pallich, sutor christianus, et Boniua Adret, sartor iudeus, minor dierum, Cervarie.

1490, juny, 30. Cervera.

Mossé Andali, jueu, denuncia l'obra nova que fa fer Bonjua Adret.

AHCC, FN, Cervera, 55, Antoni Romeu, Manual, 1489-1490, foli 87 verso.

In presencia mei dicti et infra scripti notarii et testium supra dictorum, constitutus personaliter in dicto opere d'en Boniua Adret, Mosse Andalí, undeus, dixit proiciendo tres lapides: «Jo denunciu obra nova, obra nova, obra nova, la qual remet a justícia si.s pot fer o no, requerint a vós en notari m'o continueu». Quibus, etc.

Testes predicti.

1490, juny, 30. Cervera.

Samuel Cavaller, jueu, procurador de Jucef Cavaller, pare seu, denuncia l'obra nova que fa fer Bonjua Adret.

AHCC, FN, Cervera, 55, Antoni Romeu, Manual, 1489-1490, foli 87 verso.

In presencia mei dicti et infra scripti notarii et testium supra dictorum, constitutus personaliter in dicto opere d'en Boniua Adret, Samuel Cavaller, iudeus filius luceff Cavaller, fisici, ut asertus procurator dicti sui patris, proiciendo in dictum operus tres lapides dicendo "obra nova, obra nova, obra nova", requisivit me notarium quatenus de predictis sibi facerem unum et plura, etc. 
Testes sunt proxime dicti.

1490, juny, 30. Cervera.

Bonet Bellsom, jueu de Cervera, protesta per l'obra nova que fa fer Bonjua Adret.

AHCC, FN, Cervera, 55, Antoni Romeu, Manual, 1489-1490, foli 88.

In presencia mei dicti et infra scripti notarii et dictorum testium, constitutus personaliter in dicto opere d'en Boniua Adret, Bonet Bellsom, iudeus dicte ville, dixit talia vel similia verba in effectu: «Jo protest en aquesta obra perquè és interès de la mia casa e, si per justícia se pot guanyar, que li deman des d'ara esmena de qualsevol interès que sie per la mia casa e de les obres que y he fetes". Quibus, etc.

Testes sunt predicti.

1491, juliol, 22. Cervera.

Samuel Jucef Cavaller, jueu de Cervera, procurador de Jucef Cavaller, pare seu, requereix a Berenguer Gaçó, notari de Cervera, el trasllat d'un determinat document notarial.

AHCC, FN, Cervera, 53, Antoni Bonet, Manual, 1490-1492, foli 48 verso.

Die XXII ${ }^{\mathrm{a}}$ mensis iulii, anno predicto a nativitate Domini millesimo $\mathrm{CCCC}^{0}$ nonagesimo primo.

Dicta die in presencia mei Anthonii Bonet, ville Cervarie, auctoritate regia notarii publici infra scripti, et in presencia eciam honorabilium Narcissi Ortigues, mercatoris, et Bernardi Puigsabró, paratoris lane, dicte ville, testium ad ista vocatorum specialiter et assumptorum, Samuel luceff Cavaller, iudeus ville Cervarie, filius et procurator et nomine et pro parte ac coniunta persona magistri luceff Cavaller, iudei ffisici eiusdem ville, constitutus personaliter ante domum discreti Berengarii Gaçó, notarii dicte ville, verbo dixit et exposuit, coram dicto discreto Berengario Gaçó, notario, ibidem presenti, hec vel similia verba in effectu: «Sènyer en Berenguer, jo us requir que.m doneu translat de la apel.lació interposada per lo batle de Bioscha davant lo veguer per rahó de certa marcha declarada contra ell e los sotmesos a sa juredicció, la qual vós haveu testificada. Altrament protest que no.m còrrega temps fins la agau dada».

Et continuo dictus discretus Berengarius Gaçó, notarius, respondendo premissis dixit que ell demane translat de la requesta li fa e que no li còrregue temps fins la age e que no sie clos l'acte sens resposta.

De quibus, etc., instrumentum et instrumenta, etc, tot quot, etc. 


\section{APÉNDIX /I}

VOCABULARI ${ }^{6}$

\begin{tabular}{|c|c|c|}
\hline a & casa & deure \\
\hline$a b$ & castell & deute \\
\hline absent & caure & dia \\
\hline ací & cavaller & dies \\
\hline açò & celebrar & dilluns \\
\hline acolrar & censals & dins \\
\hline acordar & certa & dir \\
\hline actes & certificar & disapte \\
\hline actor & cinch & discret \\
\hline ajustar & citació & dissapte \\
\hline al & citar & distribució \\
\hline aliama & cloure & distribuir \\
\hline als & ço & dita \\
\hline altra & $\mathrm{com}$ & domicili \\
\hline altrament & consell & donar \\
\hline altre & consentir & dret \\
\hline altres & content & dues \\
\hline anar & contents & e \\
\hline ans & continent & el (pron.) \\
\hline any & continuar & ell \\
\hline aparellar & contra & ells \\
\hline apel.blació & contracte & em \\
\hline aqueles & contribuir & emperò \\
\hline aquell & córrer & en \\
\hline aquella & coses & encubar \\
\hline aquells & creure & ens \\
\hline aquest & cullir & entendre \\
\hline aquesta & cuyta & esmena \\
\hline aquí & danys & ésser \\
\hline ara & davant & etc. \\
\hline assignar & de & exercici \\
\hline avant & declarar & $\mathrm{fe}$ \\
\hline axí & del & fer \\
\hline balle & deliberar & feynes \\
\hline batle & dels & ffe \\
\hline batre & demanar & $\mathrm{fi}$ \\
\hline bells & demunt & fill \\
\hline blats & denunciar & fins \\
\hline car & des & forma \\
\hline çarabatana & designar & forment \\
\hline càrrech & despeses & fricció \\
\hline carta & destribuir & gosar \\
\hline
\end{tabular}

${ }^{6}$ Les formes verbals, les hem passat a infinitius. por lo que no tenemos una casuística real 


\begin{tabular}{|c|c|c|}
\hline gran & magnífic & per \\
\hline guanyar & magnífich & perill \\
\hline habitació & manaments & però \\
\hline haver & manera & perquè \\
\hline he (conj.) & marcha & plaure \\
\hline hi (conj.) & marit & poder \\
\hline hi (pron.) & mas & posts \\
\hline hic & me & present \\
\hline ho & més & presentació \\
\hline hostatges & mesions & presentar \\
\hline i (pron.) & messions & presents \\
\hline ignorar & mestre & prest \\
\hline il.lustríssimo & mesurar & prestar \\
\hline infant & metre & prests \\
\hline interès & $\mathrm{mi}$ & principal \\
\hline interessos & mia & procurador \\
\hline interposar & migeres & procuradós \\
\hline intima & molt & protestar \\
\hline intimar & moltes & provehir \\
\hline io & mon & provisió \\
\hline iuxta & mossèn & pubills \\
\hline ja & mostrar & pública \\
\hline jo & mudar & qual \\
\hline jornada & murs & qualsevol \\
\hline jorns & ne & quant \\
\hline jueus & nengú & quar \\
\hline juheus & ni & que \\
\hline jurament & no & què \\
\hline juredicció & nos & qui \\
\hline justícia & nosaltres & quiscun \\
\hline la (art.) & notari & rahó \\
\hline la (pron.) & notori & reals \\
\hline lançar & nova & rehebre \\
\hline lavors & novament & rehó \\
\hline leixar & o (conj.) & remetre \\
\hline les & o (pron.) & requerir \\
\hline letra & obeir & requesta \\
\hline leuda & obligació & res \\
\hline leudar & obra & respondre \\
\hline levar & obrar & responre \\
\hline lexar & obres & rey \\
\hline $\mathrm{li}$ & obtenir & sa \\
\hline licència & offerir & saber \\
\hline lícites & ordenar & saffrà \\
\hline liurar & paga & sagrament \\
\hline lo (art.) & pagar & salaris \\
\hline lo (pron.) & paraula & salvar \\
\hline loctinent & pasar & sdevenidor \\
\hline los & pedres & se \\
\hline madona & penes & secret \\
\hline magestat & penyora & secretari \\
\hline
\end{tabular}




$\begin{array}{lll}\text { secretaris } & \text { strangers } & \text { us } \\ \text { segar } & \text { sua } & \text { vanema } \\ \text { segell } & \text { tant } & \text { vanemar } \\ \text { sens } & \text { tempas } & \text { vegades } \\ \text { senyal } & \text { tenir } & \text { veguer } \\ \text { sènyer } & \text { terme } & \text { vels } \\ \text { senyor } & \text { testificar } & \text { venema } \\ \text { senyors } & \text { testimoni } & \text { venemar } \\ \text { serta } & \text { tirar } & \text { venir } \\ \text { setmana } & \text { tocar } & \text { vila } \\ \text { seus } & \text { tornar } & \text { virtut } \\ \text { si (adv.) } & \text { tot } & \text { voler } \\ \text { si (conj). } & \text { totes } & \text { voltes } \\ \text { sinó } & \text { tots } & \text { vos } \\ \text { sobre } & \text { trametre } & \text { vós } \\ \text { solament } & \text { translat } & \text { vosaltres } \\ \text { son } & \text { trellat } & \text { vostra } \\ \text { sotmetre } & \text { tremetre } & \text { vostre } \\ \text { spay } & \text { tres } & \text { vuy } \\ \text { specialment } & \text { triga } & \text { y (pron.) } \\ \text { star } & u \text { (pron.) } & \text { ych } \\ \text { stogar } & \text { una } & \text { yo }\end{array}$

\section{APÉNDIX III}

$$
\text { NÓMINA DE JUEUS }{ }^{7}
$$

Abram Azcarell, de Lleida, 25.

Bonadona, esposa de Jucef Cavaller, 26.

* Bonafilla, esposa de Cresques Adret, 7, 8.

* Bonet Bellsom, 28, 33.

* Bonjua Adret, major, 30, 31, 32, 33.

Bonjua Adret, menor, sastre, 30, 31, 32, 33.

Bonjua Salom, 26.

* Cresques Adret, metge, 4, 7, 8, 11, 21, 25.

Daví Bendit, 20.

* Goig, vídua de Samuel [Astruch] Cavaller i mare de Jucef Cavaller, 17.

* Içach Adret, 13.

Içach de Besés, de Tàrrega, 15.

* Içach de Quercí, 19.

* Içach de Quercí, major; 2, 5, 10, 22.

7 Hem col.locat un asterisc davant el riom d'aquells jueus amb intervenció oral reflectida en els docuçments transcrits. Els números remeten als documents. 
* Içach de Quercí, menor, 1, 12.

* Içach Jacob de Quercí, metge, 25, 26, 27. Içach Mossé Sutlam, 23.

* Içach Sutlam, 24.

* Jacob de Quercí, 9, 18, 20.

* Jacob Içach de Quercí, metge, 28.

* Jafudà de Quercí, 29. Jucef Azfor, 9

* Jucef Baró Crexent, 16, 17, 23, 26, 30.

* Jucef Cavaller, fill de Samuel Astruch Cavaller, 3, 15, 16, 17, 26, 32, 34. Jucef Samarell, 11. Lobell Samarell, 8.

* Mossé Andalí, 31.

* Mossé Sutlam, 14.

* Salamó Cavaller, metge, 23.

* Salamó Cohén, 24, 25.

* Salamó Satorra, 12. Samuel Astruch Cavaller, difunt, 3, 6.

* Samuel de Lunell, metge, 3, 6, 21.

* Samuel Jucef Cavaller, metge, fill de Jucef Cavaller, 23, 32, 34. Vidal Simeon, de Santa Coloma de Queralt, 25. 